\title{
Reliability Analysis of Damaged Beam Spectral Element with Parameter Uncertainties
}

\author{
M. R. Machado and J. M. C. Dos Santos \\ Department of Computational Mechanics, University of Campinas (UNICAMP), 13083-970 Campinas, SP, Brazil
}

Correspondence should be addressed to M. R. Machado; marcelam@fem.unicamp.br

Received 12 December 2014; Accepted 24 May 2015

Academic Editor: Gang Li

Copyright (C) 2015 M. R. Machado and J. M. C. Dos Santos. This is an open access article distributed under the Creative Commons Attribution License, which permits unrestricted use, distribution, and reproduction in any medium, provided the original work is properly cited.

\begin{abstract}
The paper examines the influence of uncertainty parameters on the wave propagation responses at high frequencies for a damaged beam structure in the structural reliability context. The reliability analyses were performed using the perturbation method, FirstOrder Reliability Method (FORM), and response surface method (RSM) which were compared with Monte Carlo simulation (MCS) under the spectral element method environment. The simulated results were performed to investigate the effects of material property and geometric uncertainties on the response at high frequency modes, such as the computational efficiency of reliability methods. For the first time, the spectral element method is used in the context of reliability analysis at medium and high frequency bands applied to damage detection. It has shown the effects of parameters uncertainty on the dynamic beam response due on an impulsive load and the robustness of each method. Numerical examples in a bending vibrating beam with random parameters are performed to verify the computational efficiency of the present study.
\end{abstract}

\section{Introduction}

At medium and high frequency bands approaches like the finite element method (FEM) create large numerical models, which can require very high computational times. Statistical Energy Analysis (SEA) produces small models but without spacial variation in subsystems [1]. An alternative to these problems is the spectral element method (SEM) [2-5]. It consists in the analytical solution of the displacement wave equation in the frequency domain written in the form of a finite element. Then, a spectral element is equivalent to an infinite number of finite elements. This characteristic and the spectral domain make SEM more suitable to solve high frequency band problems. Its wave propagation formulation is also more adequate to solve damage detection problems. In the last decade, researches about damage detection concentrate on methods that use elastic wave propagation at medium and high frequencies to detect and quantify structural damage [6-9]. They use the evidence that material discontinuities, such as a crack, generate changes in the elastic waves propagating into the structure [10]. Some particular advantages of elastic wave-based damage detection methods include their capacity to propagate over significant distances and their high sensitivity to discontinuities near the wave propagation path. The presence of a structural damage introduces a local flexibility change that modifies its vibration response [11-13]. Therefore, vibration energy can be used to investigate the damaged condition of a structure. The treatment of uncertainties using spectral element method is recent $[14,15]$, and very few were made related with detection and assessment of the damage. Recently, the authors [16-18] and some other researchers [19-21] have presented works in damage detection using wave propagation in the context of uncertainty quantification and stochastic SEM model.

Engineering analysis consists in verifying and certifying that the system complies with many performance criteria, safety, and durability under different kinds of solicitation. It is well known that there are many uncertainty sources in external loads and structural parameters. Consequently, the necessity to incorporate this information in the system design is increasing. With the aim to include uncertainty in system design, reliability methods have been developed in the last few decades. First-order second-moment (FOSM), First-Order Reliability Method (FORM), and Second-Order 
Reliability Method (SORM) [22-24] are methods based on Hasofer and Lind transformation [25], which include uncertainty in the reliability analyses. Frequently, in the reliability analysis of complex structures, the limit state function cannot be expressed in a closed form. Typically, it needs to be evaluated implicitly through an approximated solution, such as a finite element method. The reliability analysis can also be carried out using Monte Carlo (MC) simulation [26]. However, $\mathrm{MC}$ requires a large number of realizations to converge, which could be an expensive technique and for large structures it may be infeasible. In order to reduce the computation time the response surface method (RSM) has been applied [27-32]. Usually, in this case, the actual limit state function is approximated by a polynomial function. Melchers and Choi $[33,34]$ present the techniques for reliability analysis of engineering structures using probability theory. In the context of damage detection and crack propagation, reliability methods were employed using approximated techniques such as boundary element method (BEM) [35], finite element method [22, 23, 33, 36], and finite element model updating with wave propagation [37]. In this paper a new contribution to the structural damage detection of a nonpropagating crack in the context of reliability methods using the spectral element method is presented.

In this study the structural damage detection problem using the the spectral element method together with reliability algorithms is presented. The damaged beam spectral element [6] is extended to include uncertainty in material property and geometric parameters. A straightforward procedure to estimate the parameter randomness is the Monte Carlo simulation [26]. It consists in obtaining a large number of deterministic analyses with different realizations of random variables. For complex structures with implicit performance function the evaluation of each realization is computationally expensive and the reliability analysis could be impracticable. Therefore, MC simulation becomes computationally infeasible to estimate the failure probability for large structures. Approximated methods like First-Order Reliability Method (FORM) and response surface method (RSM) [28] provide more appropriated tools to estimate the structural failure in these cases.

To consider probabilistic damage detection problems and random crack parameters, it is required to couple the reliability procedures with the spectral element model. This coupling can be performed using either direct coupling or response surface method. In the direct coupling the limit state function derivatives are calculated directly based on the numerical response of the SEM model [36]. In this approach, the limit state function remains implicit and is defined by the SEM model responses. The basic procedure consists in directly coupling the reliability model, FORM, with the numerical beam model based on SEM. In the reliability analysis the RSM is used to approximate the beam response at the vicinity of the most probable failure point in terms of the random variables (material properties, geometries, etc.). The beam response is estimated by a local polynomial approximations in an iterative process, as the search of the design point is evaluated [35]. Numerical tests are presented

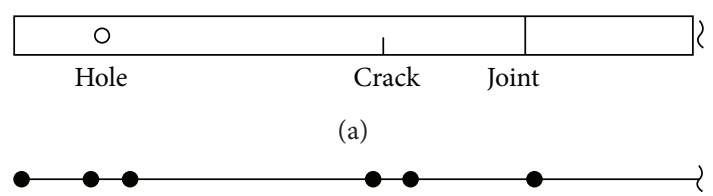

(b)

FIGURE 1: Actual structure representation (a) and spectral element model representation (b).

in order to compare the coupling of the numerical model based on SEM with reliability methods and the computational efficiency of each approach.

\section{Spectral Element Method}

The spectral element method is similar in style to the finite element method. However, there are two important differences between these methods. The first one is that SEM is a wave propagation formulation written in the frequency domain. The second one is that the element interpolation function is the exact analytical solution of the differential equation. Based on these characteristics the number of elements required for a spectral model will coincide with the number of discontinuities in the structure (Figure 1).

2.1. Beam Spectral Element. For this model it is assumed that the beam is a slender structure subjected to transversal loads and bending moments. It is also considered that transversal and rotational displacement are small. By neglecting shear deformations, the differential equation of movement in its spectral form can be written as follows [3]:

$$
\frac{d^{4} \widehat{v}}{d x^{4}}-k^{4} \widehat{v}=0
$$

with the homogeneous solution given by

$$
\begin{aligned}
\widehat{v}(x, \omega)= & A e^{-i\left(k_{1} x\right)}+B e^{-\left(k_{2} x\right)}+C e^{-i k_{1}(L-x)} \\
& +D e^{-k_{2}(L-x)}
\end{aligned}
$$

where $\widehat{v}$ is the transversal displacement in the frequency domain and $L$ is the beam length. The wave numbers, $k, k_{1}$, and $k_{2}$, are given by

$$
\begin{aligned}
& k^{2} \equiv \sqrt{\frac{\omega^{2} \rho S}{E I}}, \\
& k_{1}= \pm k \\
& k_{2}= \pm i k
\end{aligned}
$$

where $\omega$ is the circular frequency, $E$ is Young's modulus, $S$ is the cross section area, $\rho$ is the density, $I$ is the inertia moment, and $i=\sqrt{-1}$. By using a complex Young's modulus, $E_{c}=E(1+i \eta)$, an internal structural damping is introduced where $\eta$ is the hysteretic structural damping factor. Figure 2 illustrates a two-node undamaged beam spectral element 


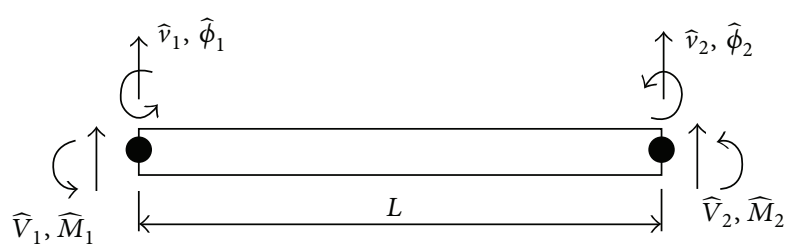

FIGURE 2: Two-node undamaged beam spectral element.

model with two degrees of freedom by nodes $(\widehat{v}$ and $\widehat{\phi})$ and nodal loads $(\widehat{V}$ and $\widehat{M})$.

By applying the displacement and rotation boundary conditions to nodes 1 and 2 (equation (2)) the coefficients, $A, B$, $C$, and $D$, can be obtained. By substituting these coefficients in (2), the expression to calculate the displacements and the rotations for any arbitrary point of the beam element can be written as

$$
\widehat{v}(x)=\widehat{g}_{1}(x) \widehat{v}_{1}+\widehat{g}_{2}(x) \widehat{\phi}_{1}+\widehat{g}_{3}(x) \widehat{v}_{2}+\widehat{g}_{4}(x) \widehat{\phi}_{2},
$$

where $\widehat{g}_{i}(x)$ are interpolation functions, which are omitted here for brevity [3]. By using the relationship between nodal loadings and displacements and applying the boundary conditions to the beam spectral element, the following matrix equation is obtained:

$$
\left\{\begin{array}{c}
\widehat{V}_{1} \\
\widehat{M}_{1} \\
\widehat{V}_{2} \\
\widehat{M}_{2}
\end{array}\right\}=[\widehat{\mathbf{K}}](\omega)\left\{\begin{array}{l}
\widehat{v}_{1} \\
\widehat{\phi}_{1} \\
\widehat{v}_{2} \\
\widehat{\phi}_{1}
\end{array}\right\}
$$

where $\widehat{\mathbf{K}}(\omega)$ is the undamaged beam spectral element dynamic stiffness matrix, which is symmetrical and usually complex and can be expressed as

$$
\widehat{\mathbf{K}}(\omega)=\frac{E I}{L^{3}}\left[\begin{array}{cccc}
1 & 1 & e^{-i k L} & e^{-k L} \\
-i k & -k & i k e^{-i k L} & k e^{-k L} \\
e^{-i k L} & e^{-k L} & 1 & 1 \\
-i k e^{-i k L} & -k e^{-k L} & i k & k
\end{array}\right] .
$$

The throw-off beam spectral element can be thought of as a particular case of the undamaged beam spectral element where node 2 on right-hand side goes to infinity (Figure 3 ). In the context of probabilistic analysis this element will be considered as a deterministic element. Following a similar procedure, the throw-off beam spectral element dynamic stiffness matrix can be obtained as

$$
\widehat{\mathbf{K}}_{t}(\omega)=\frac{E I}{L^{3}}\left[\begin{array}{cc}
(i-1) k^{3} & i k^{2} \\
i k^{2} & (i-1) k^{3}
\end{array}\right] .
$$

The damaged beam spectral element is a beam including a transverse, open, and nonpropagating crack [6] as illustrated in Figure 4. The crack is modelled by a dimensionless local flexibility, $\Theta$, which is calculated based on Castigliano's theorem and the laws of fracture mechanics [38].

The solution for (2) must be written in two parts:

$$
\begin{aligned}
\widehat{v}^{l}(x)= & A_{1} e^{-i\left(k_{1} x\right)}+B_{1} e^{-\left(k_{2} x\right)}+C_{1} e^{-i k_{1}\left(L_{1}-x\right)} \\
& +D_{1} e^{-k_{2}\left(L_{1}-x\right)} \quad\left[0 \leq x \leq L_{1}\right], \\
\widehat{v}^{r}(x)= & A_{2} e^{-i k_{1}\left(L_{1}+x\right)}+B_{2} e^{-k_{2}\left(L_{1}+x\right)}+C_{2} e^{-i k_{1}\left(L-\left(L_{1}+x\right)\right)} \\
& +D_{2} e^{-k_{2}\left(L-\left(L_{1}+x\right)\right)} \quad\left[0 \leq x \leq L-L_{1}\right],
\end{aligned}
$$

where $\widehat{v}^{l}$ and $\widehat{v}^{r}$ are the vertical displacement on the leftand right-hand side of the crack, respectively. The coefficients $A_{1}, B_{1}, C_{1}, D_{1}, A_{2}, B_{2}, C_{2}$, and $D_{2}$ are determined by the boundary conditions. Displacements, nodal loads, and the element dynamic stiffness matrix are obtained in a similar way as the undamaged beam spectral element. Then, the damaged beam spectral element dynamic stiffness matrix can be written as

$$
\widehat{\mathbf{K}}_{c}(\omega)=\frac{E I}{L^{3}}\left[\begin{array}{cccccccc}
1 & 1 & a & b & 0 & 0 & 0 & 0 \\
-i k & -k & i k a & k b & 0 & 0 & 0 & 0 \\
-a & -b- & -1 & -1 & a & b & c & d \\
i a k-a \Theta k^{2} & b k+b \Theta k^{2} & -i k-\Theta k^{2} & -k+\Theta k^{2} & -i k a & -k b & i k c & k d \\
-k^{2} a & k^{2} b & -k^{2} & k^{2} & k^{2} a & -k^{2} b & k^{2} c & -k^{2} d \\
i k^{3} a & -k^{3} b & -i k^{3} & k^{3} & -k^{3} a & k^{3} b & i k^{3} c & -k^{3} d \\
0 & 0 & 0 & 0 & f & g & 1 & 1 \\
0 & 0 & 0 & 0 & -i k f & -k g & i k & k
\end{array}\right] \text {, }
$$




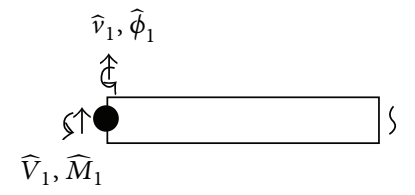

Figure 3: One-node throw-off beam spectral element.

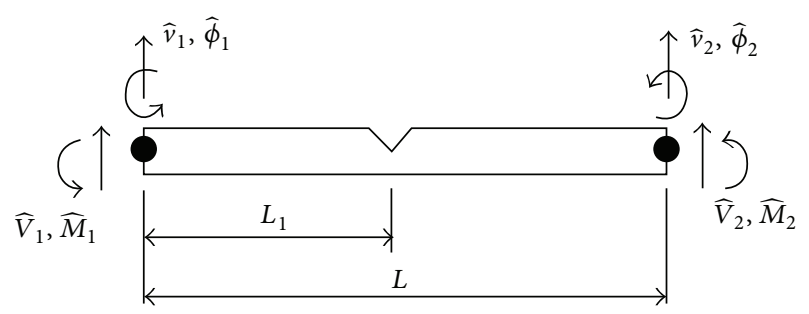

Figure 4: Two-node damaged beam spectral element.

where $a=e^{i k L_{1}}, b=e^{-k L_{1}}, c=e^{-i k\left(L-L_{1}\right)}, d=e^{k\left(L-L_{1}\right)}, f=$ $e^{-i k L}$, and $g=e^{-k L}$.

2.2. Flexibility at the Crack Position. The crack flexibility coefficient $(\Theta)$ is calculated using Castigliano's theorem, where the flexibility at the crack location for the one-dimensional beam spectral element can be obtained as

$$
c=\frac{\partial^{2} U}{\partial P^{2}}
$$

where $U$ denotes the elastic strain energy due to the crack and $P$ is the nodal force on the element.

Figure 5 shows a detail of the cross section area at the crack position. By considering that only crack mode $I$ is present in the beam element, the elastic strain energy can be expressed as

$$
U=\frac{1-v^{2}}{E} \int_{S_{c}} K_{I}^{2} d S_{c},
$$

where $\nu$ is Poisson's ratio, $S_{c}$ is the damaged cross section area, and $K_{I}$ is the stress intensity factor corresponding to the crack mode $I$, which can be written by

$$
K_{I}=\frac{6 M}{b h^{2}} \sqrt{\pi a} \mathrm{f}\left(\frac{a}{h}\right),
$$

where $b$ is the cross section base, $h$ is the cross section height, $a$ is the crack depth, $M$ is the bending moment at crack position, and $f$ is the fracture mechanics correction function given by

$$
\begin{aligned}
& f\left(\frac{a}{h}\right) \\
& \quad=\sqrt{\frac{2 h}{\pi a} \tan \left(\frac{\pi a}{2 h}\right)} \frac{0.923+0.199[1-\sin (\pi a / 2 h)]^{4}}{\cos (\pi a / 2 h)} .
\end{aligned}
$$

It can be shown that crack flexibility can be expressed as

$$
c=\frac{72 \pi}{b h^{2}} \int_{0}^{\alpha} \alpha \mathrm{f}^{2}(\alpha) d \alpha,
$$

where $\alpha=a / h$ is the dimensionless crack depth. The dimensionless local flexibility is given by

$$
\Theta=\frac{E I c}{L} .
$$

A spectral element for undamaged and damaged beam was present in this section. These models will be used to simulate the response of the structural system. Uncertainty parameters are considered in these formulations with the exception of throw-off element. It is used here only to throw off the energy of the system and has no significant influence on the dynamic system response.

\section{Coupling Reliability Analysis and SEM Modelling}

The aim of reliability analysis is to estimate the failure probability in view of a certain failure scenario $[33,34]$. The performance or safety of the structure can be described by limit state functions $g(\mathbf{X})=g\left(X_{1}, X_{2}, \ldots, X_{n}\right)$, where the parameters which are significant sources of uncertainty in the system are grouped in vector $X_{i}$. The limit state functions of interest can be defined as $g(\mathbf{X})=0$, although in real situation a random behaviour can be observed. In this case the limit state function $g(\mathbf{X})$ is separate in safe domain, where $g(\mathbf{X})>$ 0 , and in failure domain, where $g(\mathbf{X})<0$. However, the limit state function can be calculated at some desired points by running the SEM analysis. The failure probability is calculated by the integral of the limit state function as

$$
p_{f}=\int_{g(\mathbf{X}) \leqslant 0} f_{X}\left(x_{1}, x_{2}, \ldots, x_{n}\right) d x_{1} d x_{2} \cdots d x_{n} .
$$

Reliability analysis is based on probabilistic algorithms to calculate the consequence of a random input on the system response. Uncertainty quantification is performed by computing approximate response functions based on random variable probability distributions. These methods are more able to compute events with low probability than sampling based approaches, since the sampling size required to solve can be unaffordable. In order to evaluate probabilistic damage detection problems, it is required to couple the reliability procedures with the spectral element model. This coupling will be performed using direct coupling and response surface method. Different treatments of reliability procedures coupling with SEM are used to perform the reliability analysis of structural damage: FirstOrder Reliability Method (FORM), First-Order Reliability Method with limit state function obtained by response surface first-order approximation (FORM_RSM1), Monte Carlo using response surface first-order (MC_RSM1) and secondorder (MC_RSM2) approximations, and Monte Carlo (MC) simulation as reference.

3.1. Direct Coupling. The direct coupling or direct method consists in directly coupling the reliability model, FORM or MC, with the numerical model of SEM. As mentioned, the limit state function defines the interface between safe 

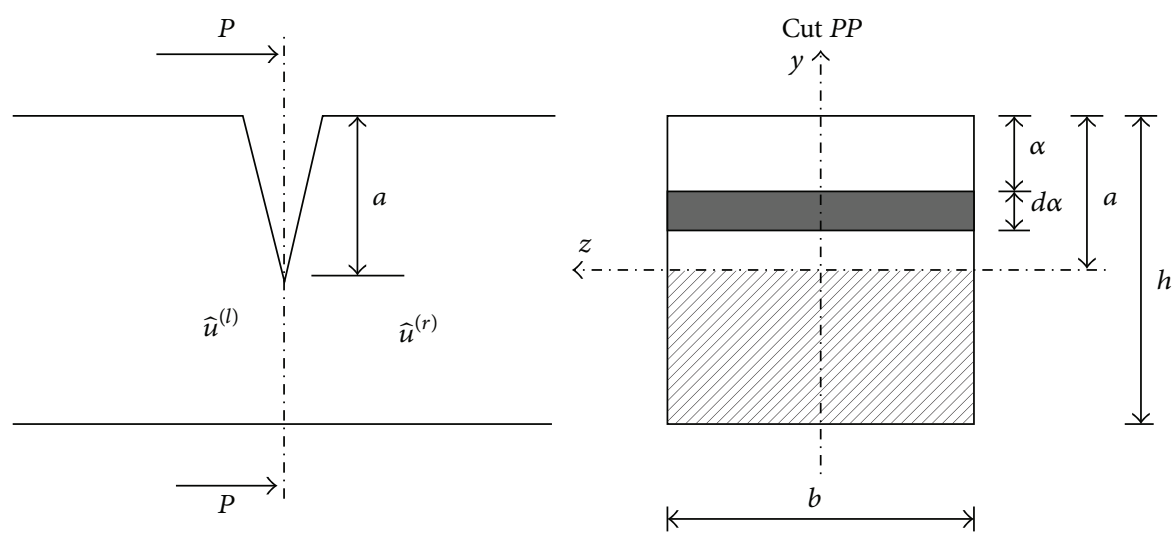

FIGURE 5: Beam cross section at the crack position.

and failure domains. In a context of structural damaged detection analysis, the limit state function is defined in function of the rms (root mean square) value of deterministic system response, $\bar{r}$, and random system response, $r(\mathbf{X})$. Both system responses derived from SEM numerical model in time domain.

When the system limit state equation or performance function is known, the FORM method can be used for reliability analysis. The limit state equation can be a linear function of correlated or uncorrelated normal variables or a nonlinear function represented by its first-order approximation. If there are nonnormal variables, the method requires to work with equivalent normal variables [23]. The development of the FORM method can be traced to second-moment methods: first-order second-moment (FOSM) and advanced first-order second-moment (AFOSM). They consider the first and the second moments of random variables, that is, the mean and standard deviation. In the structure field, a failure could be defined as a structural parameter, whose value is higher than aiven critical value. In this case we have a simple limit state function defined as

$$
g(\mathbf{X})=\bar{r}-r(\mathbf{X}),
$$

where $\bar{r}$ is the critical value of the variable $r(\mathbf{X})$ to the random variables $\mathbf{X}$. For all cases failure domain is defined as $g(\mathbf{X}) \leq 0$ and safe domain as $g(\mathbf{X})>0$. Then, the failure probability will be defined as

$$
p_{f}=\int_{\Gamma} f(\mathbf{X}) d \mathbf{X}
$$

where $f(\mathbf{X})$ is the joint probability density function of the random variables and $\Gamma$ is the region of failure $(g(\mathbf{X}) \leq 0)$. In the FORM method it is approximated by

$$
p_{f}=1-\Phi(\beta)
$$

where $\Phi$ is the cumulative distribution function of the standard normal variable and $\beta$ is the distance from the origin to the approximated hypersurface called reliability index [25, 39]. For the FORM algorithm the limit state function needs to be solved to find the new design point, although this may be difficult in the case of complex nonlinear or implicit limit state functions. An algorithm suggested by Rackwitz and Fiessler [39] can be applied to overcome this problem, which uses the derivatives to find the next iteration point.

Another direct coupling approach presented in this paper and also used as a reference is the Monte Carlo simulation. A widely used application of Monte Carlo simulation is to determine the value of a multidimensional definite integral. Let us consider that the following integral needs to be evaluated:

$$
p_{f}=\int I(\mathbf{X}) f(\mathbf{X}) d \mathbf{X} .
$$

By assuming $f(\mathbf{X})$ as a probability density function we can estimate $p_{f}$ as

$$
p_{f}=\frac{1}{N} \sum_{i=1}^{N} I\left(X_{i}\right),
$$

where the $N$ values $X_{i}$ have been sampled with density $f(\mathbf{X})$ and $I(\mathbf{X})$ is the indicator function given by

$$
I(\mathbf{X})= \begin{cases}1 & \text { if } g(\mathbf{X}) \leq 0 \\ 0 & \text { if } g(\mathbf{X})>0\end{cases}
$$

The MC approach uses the so-called Simple Random Sampling, which is quite inefficient in the majority of cases. From (21) it is obvious that the accuracy of the estimation will depend on the number of samples. The failure probability would lead to the true value as the number of samples goes to infinity. Then the sample size to accurately estimate the failure probability is a matter of concern. There are several ways to evaluate the accuracy of (21). One way is to study the error associated with the sample size by approximating the binomial distribution with the normal distribution and estimating the $95 \%$ confidence interval of the failure probability [40]. It can be shown that the sampling size with a confidence of $95 \%$ can be obtained with

$$
N=\frac{4\left(1-p_{f}\right)}{p_{f} \varepsilon^{2}} .
$$


In the reliability context, the failure probability is computed based on (22). For a certain number of samples, when the state limit equation is lower then zero $(g(\mathbf{X}) \leq 0)$ a failure is considered, and the reliability index is calculated by the first and second moment of the number of samples that was considered.

3.2. Response Surface Method (RSM). The response surface method (RSM) is a set of statistical techniques designed to find the best response value, considering the uncertainty or variations in the values of input variables [41]. This method can be employed to estimate the structural statistic response or the implicit limit state functions, which is represented by the mean and coefficient of variation of the response. In RSM, the true limit state function, $g(\mathbf{X})$, is approximated by a simple and explicit mathematical expression, which is a $n$th order polynomial with undetermined coefficients. The value of the limit state function is evaluated at a number of samples of $\mathbf{X}$, to determine the unknown coefficients such that the error of approximation at the samples of $\mathbf{X}$ is minimized. The selection form of the approximated limit state function should be based on the shape and the nonlinearity of the true limit state function. Since $g(\mathbf{X})$ is usually unknown, there has been a tendency to develop a generic form for a response surface which can be applied across a wide range of structural reliability problems. The most common forms are the first- or second-order polynomial. However, to capture the nonlinearity of the limit state function more precisely, cross terms are sometimes included into the polynomial, which can be written in a more general form as

$$
\widehat{g}(\mathbf{X})=b_{0}+\sum_{i=1}^{n} b_{i} X_{i}+\sum_{i=1}^{n} b_{i i} X_{i}^{2}+\sum_{i=1}^{n-1} \sum_{j>1}^{n} b_{i j} X_{i} X_{j},
$$

where $X_{i}(i=1,2, \ldots, k)$ are the $i$ th random variables, $n$ is the total number of random variables, $b_{0}, b_{i}, b_{i i}$, and $b_{i j}$ are the unknown coefficients to be determined, and $\widehat{g}(\mathbf{X})$ is an approximate representation of the limit state function $g(\mathbf{X})$. The values of the coefficients can be determined via regression analysis using a set of sample points from the limit state function, $g(\mathbf{X})$. The number of sample points must be larger or at least equal to the number of coefficients. Among various sampling methods, a common Design of Experiment (DOE) approach is evaluated for $g(\mathbf{X})$ at $2^{n}+1$ combinations of $\mu_{i}$ and $\mu_{i} \pm z \sigma_{i}$, where $\mu_{i}$ and $\sigma_{i}$ are the mean and standard deviation of $X_{i}$ and $h$ is an arbitrary factor. In this case, the number of sample points is just sufficient for the determination of the coefficients, by this means minimizing the number of sample points and the number of evaluations of the limit state function. The RSM can approximate the limit state function accurately for roughly linear and quadratic limit states. However, when the shape of the limit state function is not close to linear or quadratic, parameter $z$ plays an important role in the accuracy of the second-order RSM approximation [31]. In the solution of structural reliability problems RSM is used to approximate the structural response at the surroundings of the most probable failure point, in terms of input variables [42]. From that approximated structural response the failure probability can be evaluated using reliability methods. In this work Monte Carlo simulation and FORM are used to estimate the failure probability.

To compute the failure probability using RSM coupled with FORM the following procedure needs to be done. Different sets of points related with $X_{i}$ are chosen according to the DOE. The selected points are used as input to calculate the structural response by SEM. Then the structural responses are used to build the response surface approximated by a polynomial expression. The next step is to compute the limit state function in a standard normalized space (24) and to calculate the reliability index $(\beta)$, which is estimated through an optimization procedure. Finally, the failure probability is estimated according to FORM approximation from equations (18) and (19).

As mentioned, the limit state function in RSM approach is constructed using a polynomial approximation fitted according to the structural responses at some desired points. In this application the response surface is used to build the approximate state limit function based on the structural response from SEM. Since the RSM can only provide a local approximation, an iterative process to map the safe and failure domains needs to be done. This procedure is based on the construction of successive surfaces, each of them valid only in the surroundings of failure point. The failure probability is computed based on the amount of sample and when the approximate state limit equation is lower then zero $(\widehat{g}(\mathbf{X}) \leq 0)$ a failure is considered. The reliability index is calculated for the first and second moment and failure probability obtained. In order to evaluate the efficiency of these methods under the spectral element environment a numerical test will be presented in the next section.

\section{Numerical Analysis}

The first test is performed with deterministic parameters in order to evaluate the computational code to calculate the dynamic responses for the undamaged and damaged beam spectral element. The second test will verify the efficiency of coupling reliability analysis with spectral element modelling under parameter uncertainty environment.

4.1. Deterministic SEM Dynamic Response. For the numerical tests of SEM dynamic response two structures are analysed: one composed of an undamaged beam spectral element connected with a throw-off beam spectral element and the other composed of a damaged beam spectral element connected with a throw-off beam spectral element. Both structures are illustrated in Figure 6. Assuming a free-free boundary condition the structures are excited at node 2 on the right-hand side by a tone-burst impulsive force. The beam structures are made of steel $\left(E=210 \mathrm{GPa}, \rho=7850 \mathrm{~kg} / \mathrm{m}^{3}\right.$, and $\eta=0.01)$ with element length $L=4 \mathrm{~m}$ and rectangular cross section $(b=0.01 \mathrm{~m}$ and $h=0.03 \mathrm{~m})$. In regard to the crack, its position is fixed at $L_{1}=2 \mathrm{~m}$ and the crack depth will be varying from a small value to a value close to the failure limit $(a=0.02-0.28 h)$. 


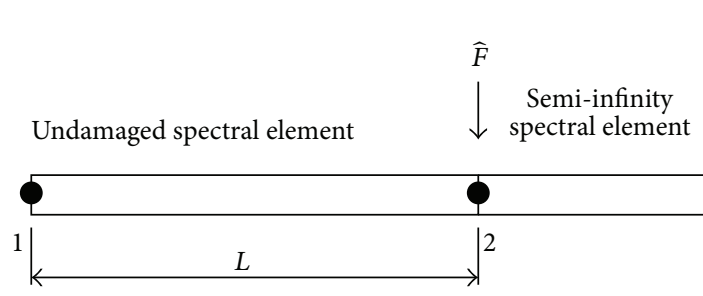

(a)

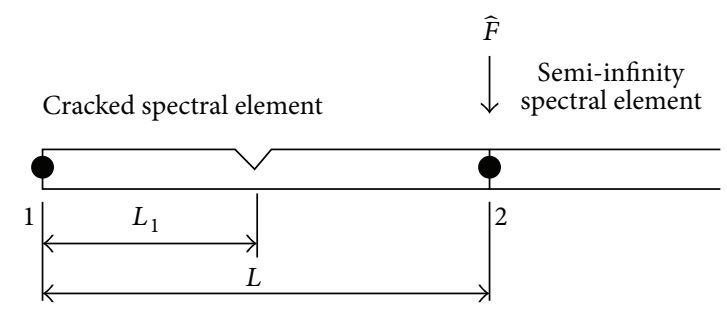

(b)

FigURE 6: Undamaged (a) and damaged (b) structural models.

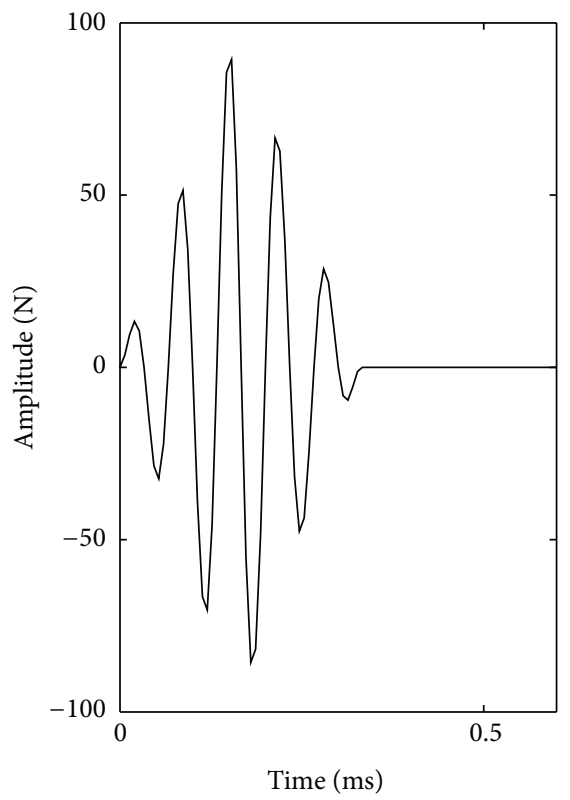

(a)

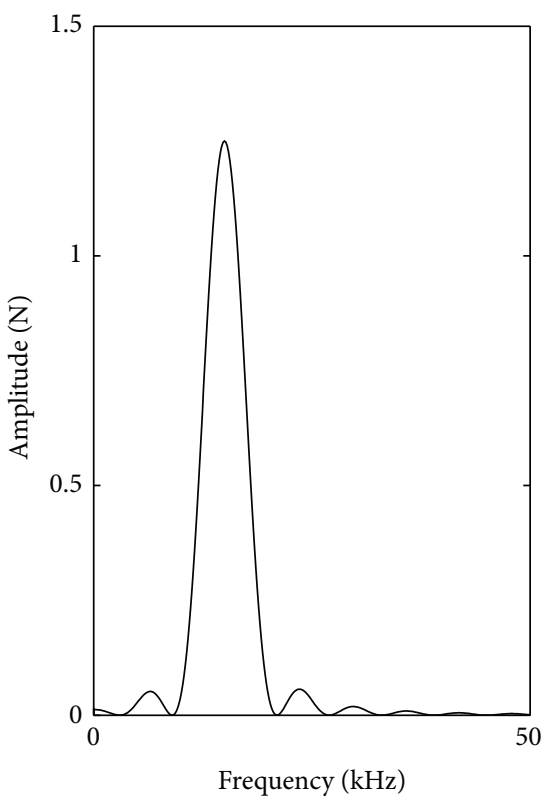

(b)

FIgURE 7: Time (a) and frequency (b) representations of tone-burst force excitation signal at $f=15 \mathrm{kHz}$ with 5 cycles.

The tone-burst impulsive force is used to improve the wave propagation visualization and to localize the crack position in the beam. Tone-burst pulse is generated using a sine signal windowed over 5 cycles with a triangular window. The sine is generated at the frequency of $15.0 \mathrm{kHz}$ with $100 \mathrm{~N}$ of amplitude. Figure 7 shows the tone-burst pulse in time and frequency domain.

For the simulated test the excitation force is applied at node 2 of the beam element, and the acceleration response is obtained at the same node. Figure 8 shows the time acceleration response for the undamaged (a) structure. It presents two pulses, one at the excitation moment (first pulse) and the other at the reflection moment (second pulse). The pulse wave travels through the structure until it reaches node 1 where it is reflected back to node 2 and continues through the throw-off element to the infinity. Throw-off element works as an anechoic termination dissipating the remaining energy into the signal. Due to structural damping $(\eta)$ the amplitude of vertical acceleration decreases through the structure length. Figure 8 shows the time acceleration response for the damaged (b) structure. The acceleration response shows two additional pulses as compared to the undamaged case, one between the excitation and reflection moments and the other after the reflection moment. These additional pulses characterize the waves partially reflected and transmitted due to any structural discontinuity, which could be a crack. These results show that the SEM model is able to reproduce the wave propagation behaviour in the undamaged and damaged beam structure and to localize the crack.

4.2. Cracked Beam Reliability Analysis. The reliability analysis will be performed for the damaged beam structure. The limit state function used to verify the structural reliability evaluates the rms value of the acceleration time response vector. It is calculated at node 2 of the beam structure $\left(a c_{2}^{(\mathrm{rms})}\right)$, which must be greater than a critical value $\left(\bar{a} c_{2}^{(r m s)}\right)$. By establishing the limit value to the nondimensional crack depth as $\alpha=0.3 \mathrm{~h}$ (30\% of beam cross section height) the critical deterministic rms value of the acceleration time response at node 2 of the 


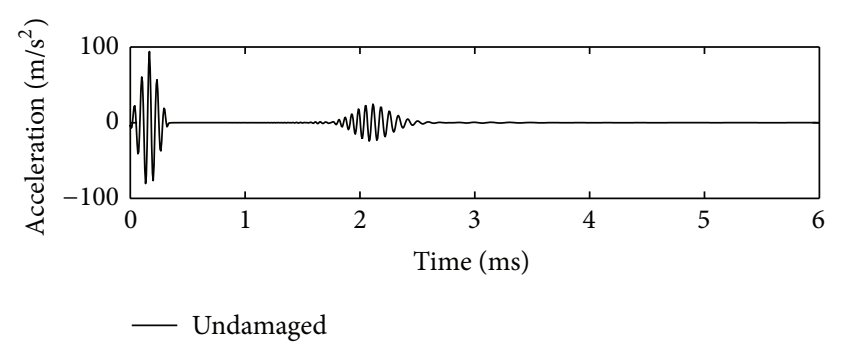

(a)

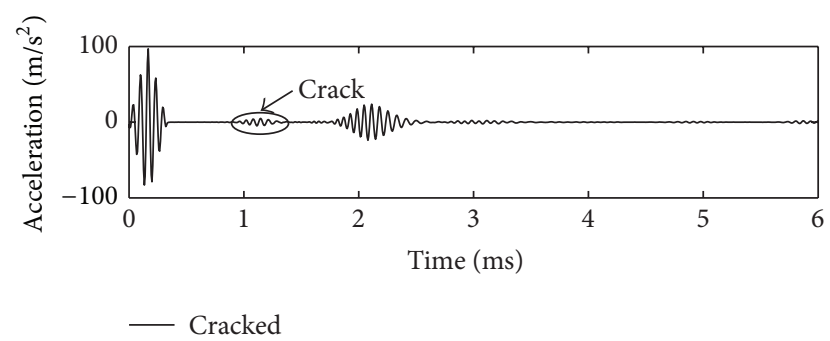

(b)

FIGURE 8: Acceleration response for undamaged (a) and damaged (b) beam.

beam structure can be obtained. From (17) the limit state function can be written as

$$
g(\mathbf{X})=\bar{a} c_{2}^{(\mathrm{rms})}-a c_{2}^{(\mathrm{rms})}(\mathbf{X})
$$

The uncertainity parameters considered are the nondimensional crack depth $(\alpha)$ with mean of $\mu_{\alpha}=[0.02: 0.08: 0.28]$ and coefficient of variation $\mathrm{COV}_{\alpha}=[0.01: 0.05: 0.31]$, Young's modulus $(E)$ with mean of $\mu_{E}=210 \mathrm{GPa}$ and $\mathrm{COV}_{E}=0.01$, crack location $L_{1}$ with $\mu_{L_{1}}=2.0 \mathrm{~m}$ and coefficient of variation of $\mathrm{COV}_{L_{1}}=0.06$, and cross section height $h$ with $\mu_{h}=$ $0.030 \mathrm{~m}$ and $\mathrm{COV}_{h}=0.06$. The random parameters are considered uncorrelated random variables with Log-normal distribution. The Log-normal distribution was assumed because the chosen random parameters cannot assume negative values. All other structural parameters are assumed as deterministic.

In order to see the effect of random parameters in the structural beam response as compared with the deterministic response a simulated test was made. Considering the specified values for the means and COV's for the structural random parameters, 100 samples of acceleration responses were calculated and compared with the deterministic response. The results are shown in Figure 9. As demonstrated, the dispersion in the parameters has a great influence in the acceleration responses. This kind of behaviour is normally seen in experimental tests. Based on this idea the random parameter is incorporated in the present study, and methods to treat this kind of randomness are used in the context of reliability analysis. By taking the crack depth with $\mu_{\alpha}=$ 0.10 and $\mathrm{COV}_{\alpha}=0.1$ and keeping all other random and deterministic variables as defined, a Monte Carlo simulation is performed to evaluate the convergence of the failure probability. Figure 10 shows that failure probability convergence can be achieved with a sample size $>500,000$. By using (23)

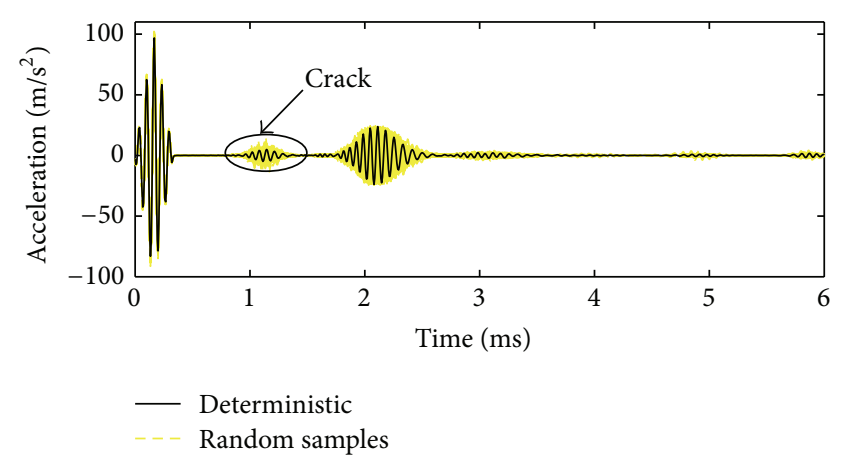

FIGURE 9: Comparison between deterministic and random system response.

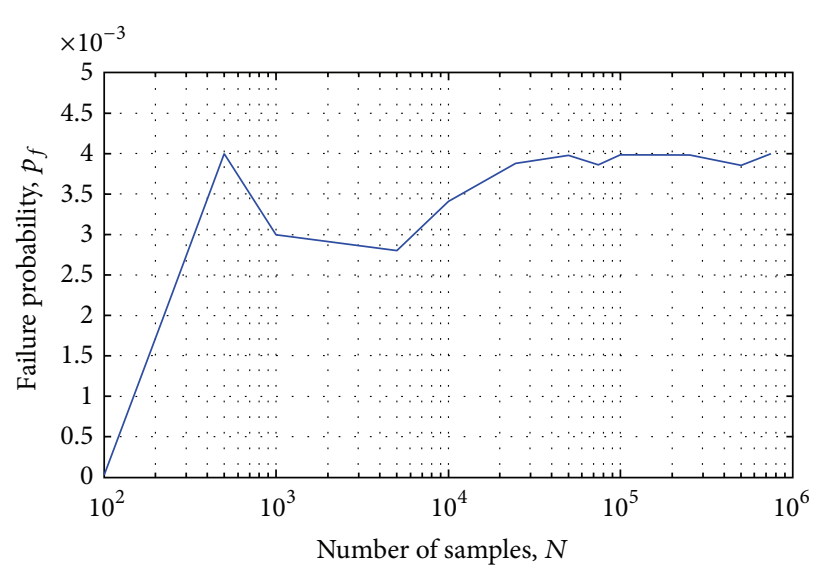

FIGURE 10: Failure probability MC convergence test.

with the converged failure probability, $p_{f} \cong 0.038$, and a $5 \%$ error $N \cong 40,000$ samples are obtained. Therefore, from now on a sampling size of 50,000 samples will be taken for all Monte Carlo simulations along the paper.

To evaluate performance and computation time to estimate failure probability using the presented methods, the following simulations are carried out. The reference for the failure probability is calculated by Monte Carlo simulation with SEM structural damaged beam model and is noted as MC. Failure probability computed by RSM first-order approximation uses full factorial DOE. It considers four random variables $\left(\alpha, E, L_{1}, h\right)$ with the levels $\mu, \mu \pm \sigma$, and evaluates the performance function for all possible combinations $\left(N_{d}=2^{n}+1=2^{4}+1=17\right.$ design points). By using (24) the coefficients for the first-order polynomial are estimated using regression analysis. For the RSM secondorder approximation the Box-Behnken design (BBD) of experiments is used. The BBD is an independent quadratic design in that it does not contain an embedded factorial or fractional factorial design. In this design the treatment combinations are at the edges midpoints of the process space and at the centre. These designs are rotated or near rotated and require three levels of each variable. For this case we consider the four random variables $\left(\alpha, E, L_{1}, h\right)$ with the levels $\mu$ and 
$\mu \pm \sigma$. The limit state function is calculated for the secondorder model without cross terms; then $p=(n+1)(n+2) / 2=$ 15 coefficients and $N_{d}=27$ design points. From (24) the coefficients for the second-order polynomial are estimated using regression analysis. When the failure probability is computed with Monte Carlo simulation with first- and second-order RSM approximation the model is referred to as MC_RSM1 and MC_RSM2, respectively. The FORM with RSM firstorder approximation works out the failure probability using the derivative of limit state function computed by finite difference method. The limit state function is approximated by a polynomial and is referred to as FORM_RMS1. The direct coupling which is the FORM coupled directly with SEM is called FORM. In order to evaluate the sensitivity of damaged beam spectral element response related with the crack depth, all simulations are performed, varying the crack depth from a small value to a value close to the allowed failure limit $(\alpha=$ $\{0.02,0.10,0.18,0.22,0.26,0.28\} h)$. Moreover, for each case, changes in crack depth coefficient of variation are made with $\mathrm{COV}_{\alpha}=\{0.01: 0.05: 0.31\}$. Figure 11(a) shows the failure probability calculated by FORM coupled directly with SEM model. The results show that for crack depth values in the range of $\mu_{\alpha}=[0.02-0.18]$ the probabilities of failure are very small, even for the highest values of $\mathrm{COV}_{\alpha}$. As the crack depth values increase to $\mu_{\alpha}=0.22$ the failure probability starts to raise as soon as the $\mathrm{COV}_{\alpha}$ value starts to increase.

For $\mu_{\alpha} \geq 0.26$ the failure probability rises in a very fast rate as the $\mathrm{COV}_{\alpha}$ value increases. Figure 11(b) shows the failure probability calculated by FORM_RSM1. The results show that for crack depth values in the range of $\mu_{\alpha}=[0.02-0.18]$ the probabilities of failure are very small as observed in the direct method. As the crack depth values increase to $\mu_{\alpha}=0.22$ the failure probability starts to raise as soon as the $\mathrm{COV}_{\alpha}$ value starts to increase. However, in the limit state function approximated by a surface response function the failure probability can take a smooth increasing with the highest COVs than that obtained with direct FORM.

For $\mu_{\alpha} \geq 26$ the failure probability rises in a very fast rate as the $\mathrm{COV}_{\alpha}$ value increases. Figure 11(c) shows the failure probability calculated by MC_RSM1. The failure probability results present a similar behaviour as that of FORM_RSM1.

Figure 11(d) shows the failure probability calculated by MC_RSM2. For this case the general behaviour seems to be similar to the previous cases in that $p_{f}$ increases as $\mu_{\alpha}$ and $\mathrm{COV}_{\alpha}$ increase. Nevertheless, the failure probability starts to raise earlier. When the crack depth value $\mu_{\alpha} \geq 0.18$ and $\mathrm{COV}_{\alpha}$ increases $p_{f}$ starts to raise, but in a slower rate than the previous cases.

Figure 11(e) shows the failure probability calculated by MC, which presents a similar behaviour as MC_RSM2. The general behaviour of these results seems to be in agreement with the physic of the problem, since the probabilities of failure are very small for $\mu_{\alpha} \leq 0.10$ for all $\mathrm{COV}_{\alpha}$ 's values and become higher for $\mu_{\alpha} \geq 0.18$ at high values of $\mathrm{COV}_{\alpha}$.

In order to make the result analysis more clear, two additional plots comparing the results of all methods together are presented. In the first the low probabilities of failure (Figure 12(a)) are grouped and in the second the high probabilities of failure (Figure 12(b)) are grouped. Figure 12(a)
TABLE 1: CPU time.

\begin{tabular}{lc}
\hline Method & CPU time $[\mathrm{s}]$ \\
\hline FORM_RSM1 & 3.1 \\
FORM & 8.79 \\
MC_RSM1 & 96.3 \\
MC_RSM2 & 99.2 \\
MCS & $12,161.2$ \\
\hline
\end{tabular}

shows that low probabilities of failure obtained by MC_RSM1, FORM_RSM1, and FORM present a good agreement for all values of crack depth in this range $\left(\mu_{\alpha}=0.02-0.18\right)$. By comparing direct Monte Carlo (MC) simulation with MC_RSM1, FORM_RSM1, and FORM a significant difference appears, which means that the nonlinearities in the damaged beam spectral element do not allow a linear approximation for the state limit function. This can be confirmed by the good agreement among Monte Carlo (MC) simulation, FORM, and Monte Carlo with a second-order response surface (MC_RSM2). Figure 12(b) shows that for high probabilities of failure a similar behaviour is observed.

Monte Carlo with first- and second-order response surface (MC_RSM1 and MC_RSM2) presents very close computational times that are much lower than Monte Carlo simulation (MCS) (Table 1). FORM shows a computational time of less than $10 \%$ of MC_RSM1 and MC_RSM2. FORM with linear response surface (FORM_RSM1) presents the lowest computation time. This reduced computation time of FORM_RSM1 as compared with FORM comes from the limit state equation that must be calculated by the last approach. Moreover, stable numerical models (as SEM) lead to smooth and faster convergence of FORM, which makes this approach more efficient than other reliability algorithms as Monte Carlo simulation and response surface method. All runs were performed in a Desktop-PC using the processor Intel Core i7 CPU $930 @ 2.80 \mathrm{GHz}, 12.0 \mathrm{~Gb}$ RAM memory, and Windows 7,64 bits as the operating system.

\section{Conclusion}

The wave propagation responses in a damaged beam structure including uncertainties in material and geometric parameters are analysed. A reliability analysis is made to quantify the failure probability of a damaged structure modelled with beam spectral element at high frequencies. The limit state function evaluates the rms value of the acceleration time response which must be above the limit value. A crack depth of $\alpha=0.30$ cross section height defines the allowed deterministic rms value of the acceleration time response. By varying crack depth $\left(\mu_{\alpha}\right)$ and its coefficient of variation $\left(\mathrm{COV}_{\alpha}\right)$ the reliability analysis is performed using linear response surface method (RSM1) solved by Monte Carlo simulation (MC_RSM1) and First-Order Reliability Method (FORM_RSM1). Also, a quadratic response surface method (RSM2) is solved by Monte Carlo simulation (MC_RSM2) and all methods are compared with Monte Carlo (MC) simulation. In general, all methods present results in agreement with the physics of the problem; that is, the probabilities 


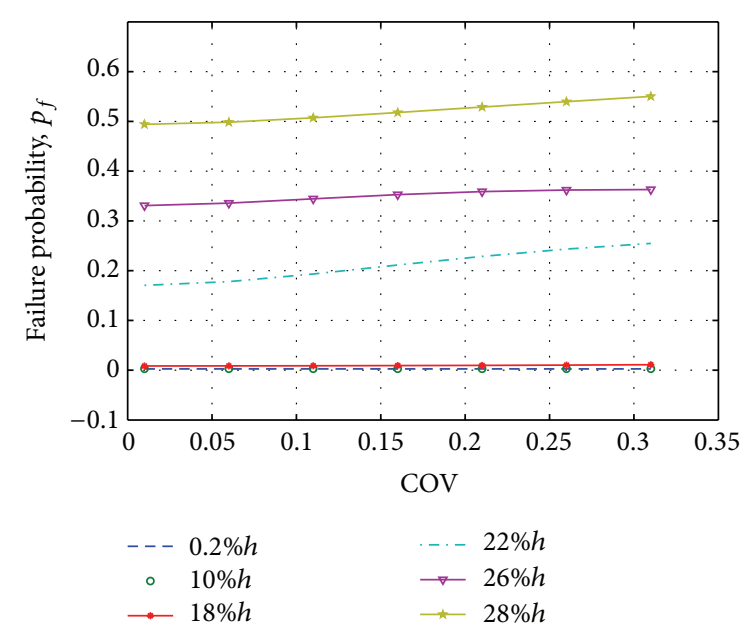

(a)

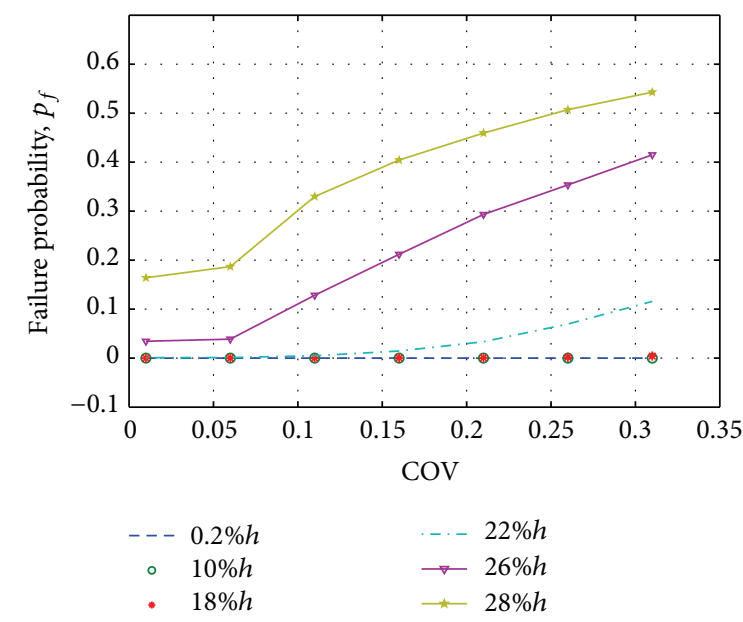

(c)
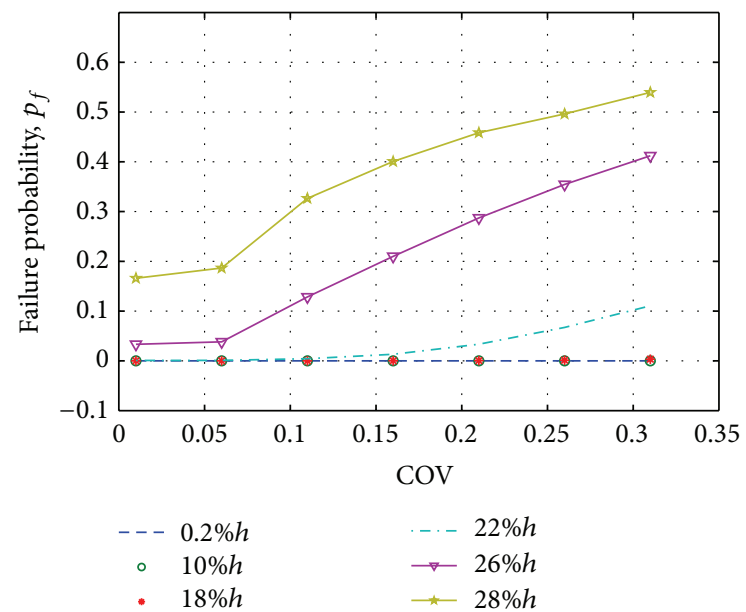

(b)
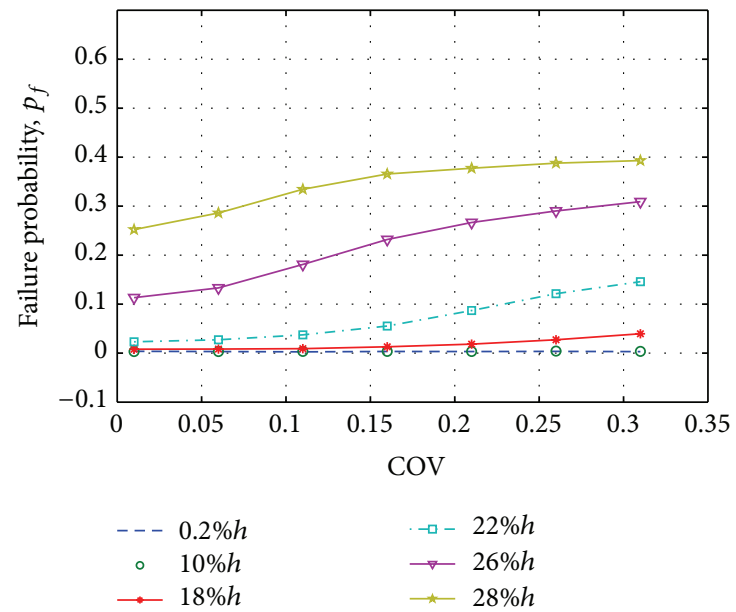

(d)

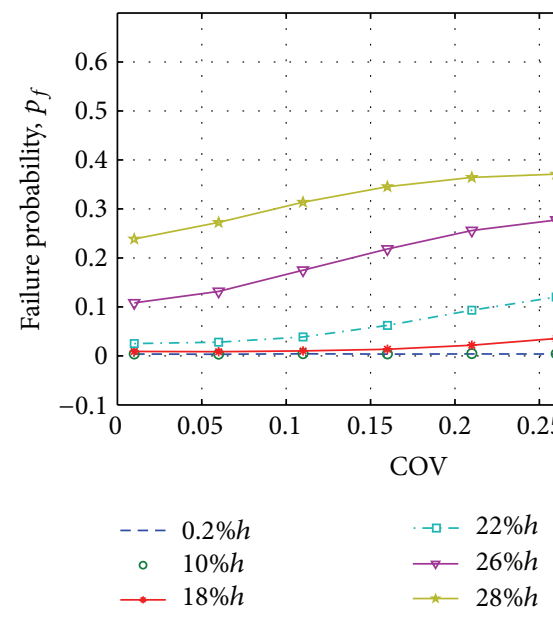

(e)

FIGURE 11: Failure probability by (a) FORM, (b) FORM with RSM first-order, (c) MC with RSM first-order, (d) MC with RSM second-order, and (e) MC. 


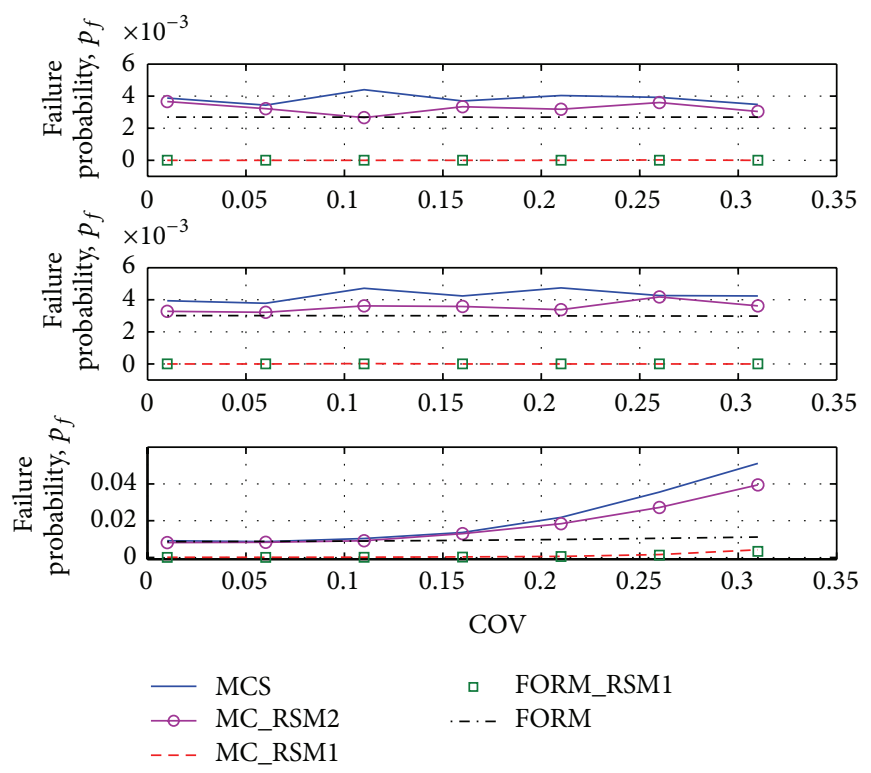

(a) Low $p_{f}$
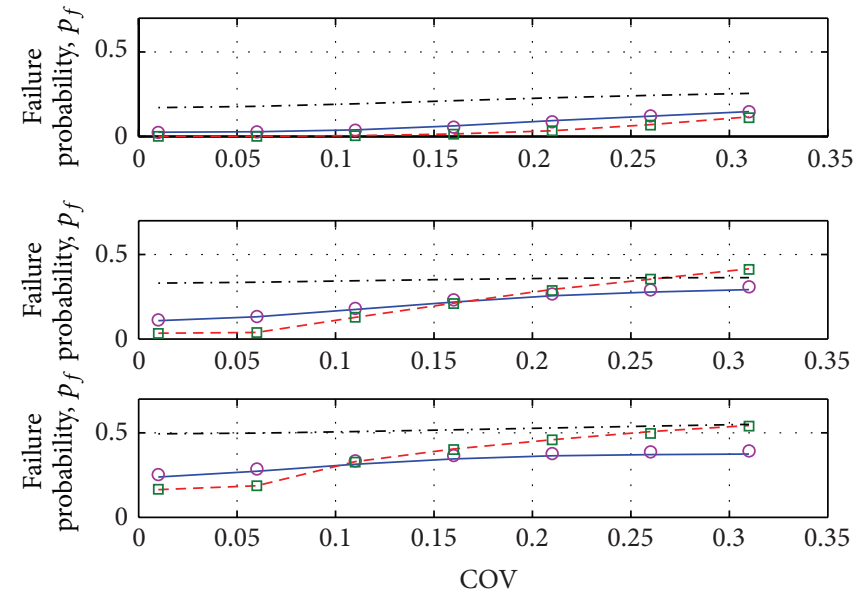

o MC_RSM2

- - MC_RSM1

(b) High $p_{f}$

Figure 12: Comparison among MCS, MC_RSM1, MC_RSM2, FORM_RSM1, and FORM at (a) low and (b) high failure probability.

of failure are very small for small crack depth and become higher as $\mu_{\alpha}$ increases. The agreement between MC_RSM1 and FORM_RSM1 confirms other literature results that a linear RSM is equivalent to computing the FORM solution directly. The disagreement between Monte Carlo (MC) and first-order RSM models (MC_RSM1 and FORM_RSM1) reveals that the degree of nonlinearity in the damaged beam spectral element does not allow a linear approximation for the state limit function. This can be confirmed by the good agreement between Monte Carlo (MC) results and Monte Carlo with a second-order Response Surface (MC_RSM2). As expected computational time results confirm that Response Surface approach reduces meaningfully the computational time as compared with direct Monte Carlo simulation. In this case Monte Carlo with second-order response surface (MC_RSM2) represents a reduction of $12,159.3 \%$ in computational time as compared with Monte Carlo (MC).

\section{Conflict of Interests}

The authors declare that there is no conflict of interests regarding the publication of this paper.

\section{References}

[1] R. H. Lyon and R. G. DeJong, Theory and Application of Statistical Energy Analysis, Butterworth-Heinemann, Boston, Mass, USA, 1995.

[2] J. F. Doyle, Wave Propagation in Structures, Springer, New York, NY, USA, 1989.

[3] J. F. Doyle, Wave Propagation in Structures: Spectral Analysis Using Fast Discrete Fourier Transforms, Mechanical Engineering Series, Springer, New York, NY, USA, 2nd edition, 1997.
[4] U. Lee, Spectral Element Method in Structural Dynamics, BInha University Press, 2004.

[5] S. Gopalakrishnan, A. Chakraborty, and D. R. Mahapatra, Spectral Finite Element Method, Springer, New York, NY, USA, 2007.

[6] M. Krawczuk, "Application of spectral beam finite element with a crack and iterative search technique for damage detection," Finite Elements in Analysis and Design, vol. 38, no. 6, pp. 537548, 2002.

[7] M. Krawczuk, J. Grabowska, and M. Palacz, "Longitudinal wave propagation. Part I-comparison of rod theories," Journal of Sound and Vibration, vol. 295, no. 3-5, pp. 461-478, 2006.

[8] W. M. Ostachowicz, "Damage detection of structures using spectral finite element method," Computers \& Structures, vol. 86, no. 3-5, pp. 454-462, 2008.

[9] Z. Su and L. Ye, Identification of Damage Using Lamb Waves, Springer, New York, NY, USA, 2009.

[10] E. R. O. Santos, J. R. F. Arruda, and J. M. C. Dos Santos, "Modeling of coupled structural systems by an energy spectral element method," Journal of Sound and Vibration, vol. 316, no. 1-5, pp. 1-24, 2008.

[11] A. K. Pandey, M. Biswas, and M. M. Samman, "Damage detection from changes in curvature mode shapes," Journal of Sound and Vibration, vol. 145, no. 2, pp. 321-332, 1991.

[12] A. K. Pandey and M. Biswas, "Damage detection in structures using changes in flexibility," Journal of Sound and Vibration, vol. 169, no. 1, pp. 3-17, 1994.

[13] A. D. Dimarogonas, "Vibration of cracked structures: a state of the art review," Engineering Fracture Mechanics, vol. 55, no. 5, pp. 831-857, 1996.

[14] S. Adhikari and M. I. Friswell, "Distributed parameter model updating using the Karhunen-Loève expansion," Mechanical Systems and Signal Processing, vol. 24, no. 2, pp. 326-339, 2010.

[15] V. Ajith and S. Gopalakrishnan, "Spectral element approach to wave propagation in uncertain beam structures," Journal of 
Mechanics of Materials and Structures, vol. 5, no. 4, pp. 637-659, 2010.

[16] A. T. Fabro, T. G. Ritto, R. Sampaio, and J. R. F. Arruda, "Stochastic analysis of a cracked rod modeled via the spectral element method," Mechanics Research Communications, vol. 37, no. 3, pp. 326-331, 2010.

[17] C. T. Ng, M. Veidt, and H. F. Lam, "Probabilistic damage characterisation in beams using guided waves," Procedia Engineering, vol. 14, pp. 490-497, 2011.

[18] E. B. Flynn, M. D. Todd, P. D. Wilcox, B. W. Drinkwater, and A. J. Croxford, "Maximum-likelihood estimation of damage location in guided-wave structural health monitoring," Proceedings of the Royal Society A, vol. 3, pp. 1-22, 2011.

[19] M. Machado and J. DosSantos, "Stochastic analysis ofwave propagation in a cracked rod via spectral element and polinomial chaos expansion," in Proceedings of the Innovations in Wave Modelling (Innowave '12), Nottingham,UK, 2012.

[20] M. R. Machado and J. M. C. dos Santos, "Damage detection in an energy flow model including parameter uncertainty," in Topics in Modal Analysis, Volume 7: Proceedings of the 31st IMAC, A Conference on Structural Dynamics, 2013, Conference Proceedings of the Society for Experimental Mechanics Series, pp. 131-140, Springer, New York, NY, USA, 2014.

[21] M. Machado, S. Adhikari, and J. DosSantos, "Damage characterization in structures with random properties," in Proceedings of the 2nd International Symposium on Uncertainty Quantification and Stochastic Modeling, Rouen, France, 2014.

[22] A. Haldar and S. Mahadevan, Reliability Assessment Using Stochastic Finite Element Analysis, John Wiley \& Sons, New York, NY, USA, 2000.

[23] S. Mahadevan and A. Haldar, Probability, Reliability and Statistical Methods in Engineering Design, John Wiley \& Sons, 2000.

[24] A. der Kiureghian, "The geometry of random vibrations and solutions by form and sorm," Probabilistic Engineering Mechanics, vol. 15, no. 1, pp. 81-90, 2000.

[25] A. M. Hasofer and N. C. Lind, "Exact and invariant second moment code format," Journal of Engineering Mechanics Division, vol. 100, no. 1, pp. 111-121, 1974.

[26] I. M. Sobol', A Primer for the Monte Carlo Method, CRC Press, 1994.

[27] L. Faravelli, "Response-surface approach for reliability analysis," Journal of Engineering Mechanics, vol. 115, no. 12, pp. 2763-2781, 1989.

[28] C. G. Bucher and U. Bourgund, "A fast and efficient response surface approach for structural reliability problems," Structural Safety, vol. 7, no. 1, pp. 57-66, 1990.

[29] M. R. Rajashekhar and B. R. Ellingwood, "A new look at the response surface approach for reliability analysis," Structural Safety, vol. 12, no. 3, pp. 205-220, 1993.

[30] K. Breitung and L. Faravelli, "Log-likelihood maximization and response surface in reliability assessment," Nonlinear Dynamics, vol. 5, no. 3, pp. 273-285, 1994.

[31] X. L. Guan and R. E. Melchers, "Effect of response surface parameter variation on structural reliability estimates," Structural Safety, vol. 23, no. 4, pp. 429-444, 2001.

[32] K. Breitung and L. Faravelli, "Response surface methods and asymptotic approximations," in Mathematical Models for Structural Reliability Analysis, CRC Press, Boca Raton, Fla, USA, 1996.

[33] R. E. Melchers, Structural Reliability Analysis and Prediction, John Wiley \& Sons, New York, NY, USA, 2nd edition, 1999.
[34] S. T. Choi, R. V. Grandhi, and R. A. Canfield, Reliability-based Structural Design, Springer, 2007.

[35] E. D. Leonel, A. T. Beck, and W. S. Venturini, "On the performance of response surface and direct coupling approaches in solution of random crack propagation problems," Structural Safety, vol. 33, no. 4-5, pp. 261-274, 2011.

[36] M. Pendola, A. Mohamed, M. Lemaire, and P. Hornet, "Combination of finite element and reliability methods in nonlinear fracture mechanics," Reliability Engineering and System Safety, vol. 70, no. 1, pp. 15-27, 2000.

[37] W.-X. Ren and H.-B. Chen, "Finite element model updating in structural dynamics by using the response surface method," Engineering Structures, vol. 32, no. 8, pp. 2455-2465, 2010.

[38] H. Tada, P. Paris, and G. R. Irwin, Stress Analysis of Cracks Handbook, Del Research Corporatoin, 1973.

[39] R. Rackwitz and B. Fiessler, "Note on discrete safety checking when using non-normal stochastic models for basic variables," Tech. Rep., MIT, Cambridge, Mass, USA, 1976.

[40] M. L. Shooman, Probabilistic Reliability: An Engineering Approach, McGraw-Hill, 1968.

[41] A. Khuri and J. A. Cornell, Response Surface: Designs and Analyses, Marcel Dekker, New York, NY, USA, 1996.

[42] E. D. Leonel, A. Chateauneuf, and W. S. Venturini, "Probabilistic crack growth analyses using a boundary element model: applications in linear elastic fracture and fatigue problems," Engineering Analysis with Boundary Elements, vol. 36, no. 6, pp. 944-959, 2012. 

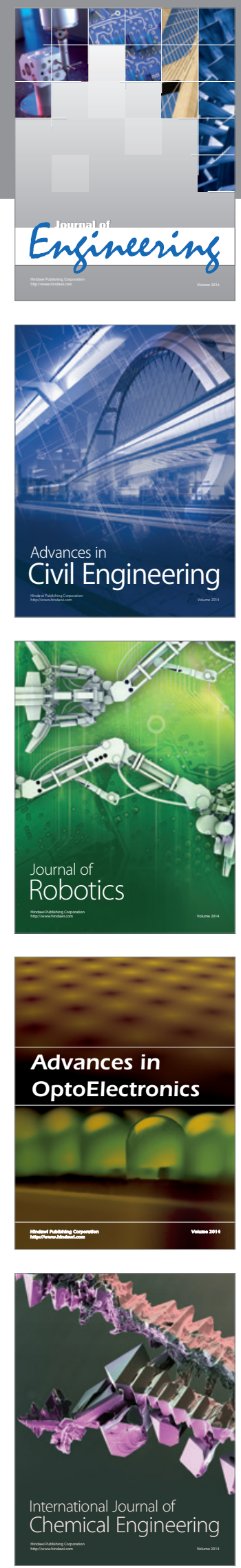

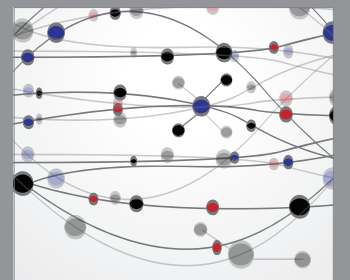

The Scientific World Journal
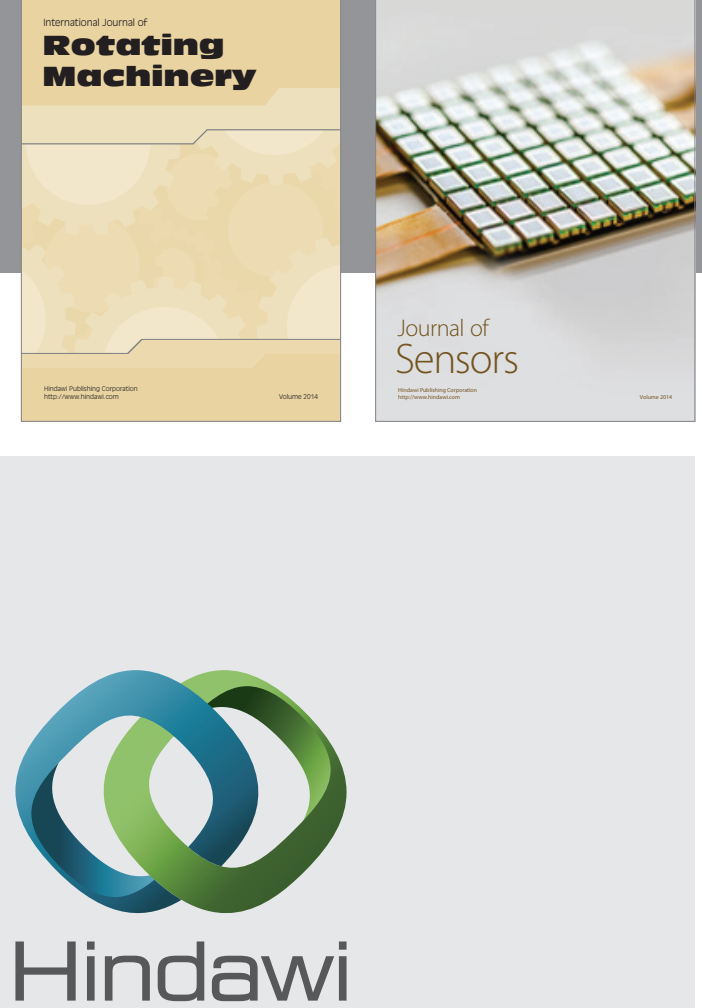

Submit your manuscripts at http://www.hindawi.com
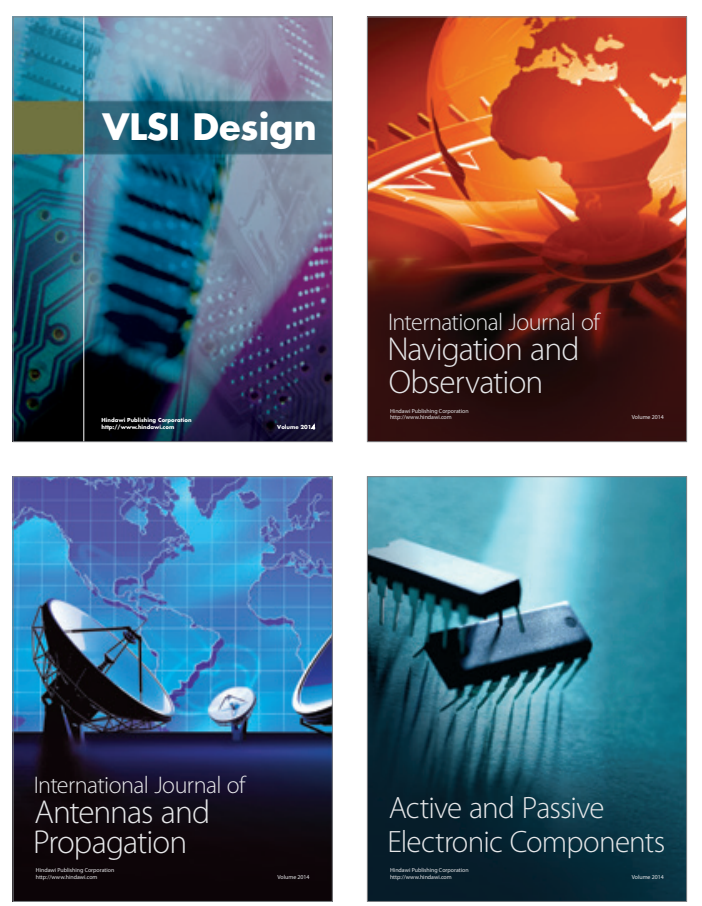
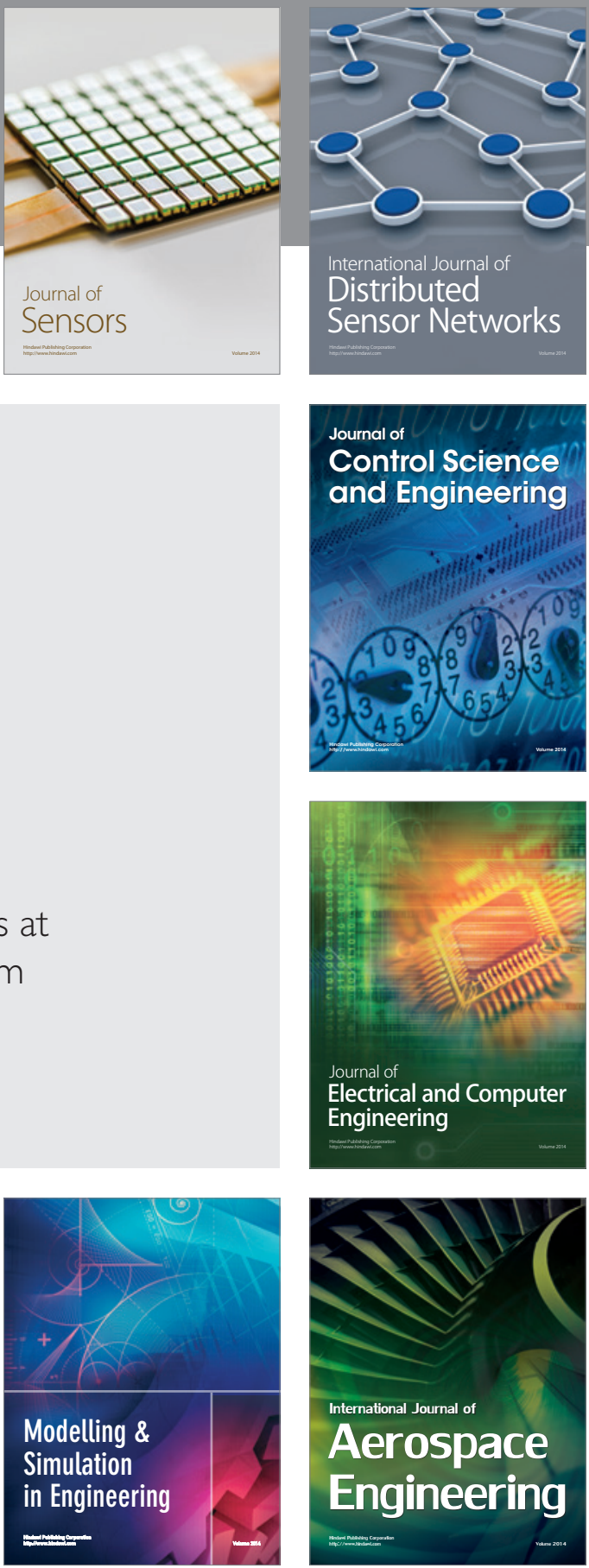

Journal of

Control Science

and Engineering
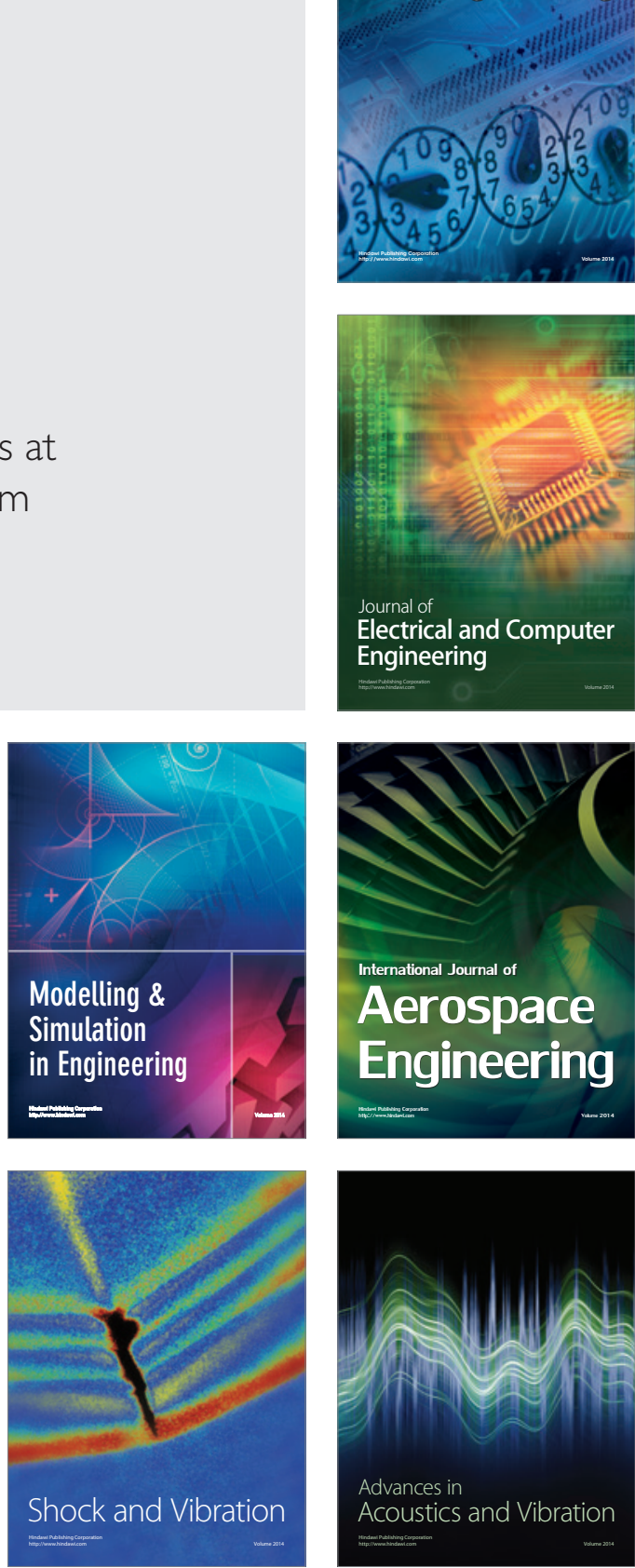